\title{
Pentafluorophenyl Esters as Exchangeable Stoppers for the Construction of Photoactive [2] rotaxanes
}

\author{
Marine Rémy, ${ }^{[a]}$ Iwona Nierengarten, ${ }^{[a]}$ Boram Park, ${ }^{[a]}$ Michel Holler,,${ }^{[a]}$ Uwe Hahn, ${ }^{[a]}$ and Jean-François \\ Nierengarten ${ }^{*[a]}$
}

Dedicated to Prof. Didier Astruc on the occasion of his $75^{\text {th }}$ birthday

[a] M. Rémy, Dr. I. Nierengarten, B. Park, Dr. M. Holler, Dr. U. Hahn, Dr. J.-F. Nierengarten Laboratoire de Chimie des Matériaux Moléculaires

Université de Strasbourg et CNRS (UMR 7402 LIMA), Ecole Européenne de Chimie, Polymères et Matériaux

25 rue Becquerel, 67087 Strasbourg Cedex 2, France

E-mail: nierengarten@unistra.fr

Supporting information for this article is given via a link at the end of the document.

\begin{abstract}
Stable pillar[5]arene-containing [2]rotaxane building blocks with pentafluorophenyl ester stoppers have been efficiently prepared on a multi-gram scale. Reaction of these building blocks with various nucleophiles gave access to a wide range of [2]rotaxanes with amide, ester or thioester stoppers in good to excellent yields. The rotaxane structure is fully preserved during these chemical transformations. Actually, the addition-elimination mechanism at work during these transformations totally prevents the unthreading of the axle moiety of the mechanically interlocked system. The stopper exchange reactions were optimized both in solution and under mechanochemical solvent-free conditions. While amide formation is more efficient in solution, the solvent-free conditions are more powerful for the transesterification reactions. Starting from a fullerene-functionalized pillar[5]arene derivative, this new strategy gave easy access to a photoactive [2]rotaxane incorporating a $\mathrm{C}_{60}$ moiety and two Bodipy stoppers. Despite the absence of covalent connectivity between the Bodipy and the fullerene moieties in this photoactive molecular device, efficient through space excited state interactions have been evidenced in this rotaxane.
\end{abstract}

\section{Introduction}

Mechanically interlocked molecules (MIMs) played a key role in the development of molecular machines. ${ }^{[1-2]}$ On the other hand, such structures are also appealing scaffolds for the construction of photoactive molecular devices in which complementary chromophores are associated into a single molecular ensemble but without being connected through covalent chemical bonds. ${ }^{[3-}$

${ }^{9]}$ As a result, intercomponent photochemical events observed in such systems occur exclusively through space. ${ }^{[3-9]}$ Sauvage was a pioneer in this particular field and his team has reported spectacular photoactive MIMs mimicking the ultra-fast charge separation observed in the natural photosynthetic system through the peculiar orientation of complementary porphyrinic moieties in rotaxanes and catenanes constructed by a metaltemplated approach. ${ }^{[3-4]}$ The same construction principle has been also intensively used to prepare related photoactive fullerene-containing systems. ${ }^{[5-6]}$ Further examples of photoactive MIMs in which through-space photoinduced electron or energy transfer events have been evidenced were also prepared by using other synthetic approaches. ${ }^{[7]}$ As part of this research, we have recently shown that pillar[5]arene-containing rotaxanes are attractive scaffolds for the construction of photoactive MIMs. ${ }^{[8-9]}$ Their synthesis was however particularly difficult and new efficient methods for the preparation of pillar[5]arene-containing rotaxanes are needed to further develop this particular field. This prompted us to develop synthetic methodologies based on stopper exchange reactions from rotaxane building blocks incorporating 2,4-dinitrophenyl ester or 3,5-bis(trifluoromethyl) benzenesulfonate stoppers. ${ }^{[10-12]}$ While very efficient for the preparation of rotaxanes incorporating an ethoxy functionalized pillar[5]arene moiety, major limitations of these building blocks are related to their moderate stability in particular on silica gel. As a result, their purification is often challenging. In order to solve this stability issue, we decided to further explore this strategy with new exchangeable stoppers. In this paper, we now report the preparation of pillar[5]arene-containing rotaxanes with pentafluorophenyl ester stoppers. These building blocks have been obtained in almost quantitative yields and are perfectly stable on silica gel thus allowing easy purifications. When compared to the previous building block with 2,4-dinitrophenol leaving groups, ${ }^{[10]}$ the stopper exchange is not only limited to the reaction with amines to afford rotaxanes with amide stoppers. Effectively, rotaxanes with ester or thioester stoppers have been also efficiently prepared by treatment of the pentafluorophenyl ester derivatives with alcohol, phenol or thiol reagents. Moreover, all the reported chemical transformations could be achieved in solution but also under mechanochemical solvent-free conditions. Finally, this new methodology has been successfully applied to the construction of a photoactive MIM incorporating a fullerene-functionalized pillar[5]arene derivative.

\section{Results and Discussion}

Preparation of the [2]rotaxane building block. Pillar[5]arenecontaining rotaxane building blocks incorporating activated ester stoppers are attractive building blocks for the preparation of a large variety of rotaxanes. We have recently shown that 2,4dinitrophenyl esters are well suited for such a purpose. ${ }^{[10]}$ However, partial hydrolysis of the 2,4-dinitrophenol ester functions was observed on silica gel and purification of the 
rotaxane building blocks was only possible by recrystallisation. This strategy was efficient when ethoxypillar[5]arene was used as starting material but the preparation of related rotaxanes from more sophisticated pillar[5]arene derivatives was found difficult. It was thus decided to explore alternative activated stoppers for the construction of pillar[5]arene-containing rotaxanes. Pentafluorophenyl ester derivatives attracted our attention. Owing to their easy preparation and their good stability, such derivatives are widely used to activate carboxylic acids for the efficient preparation of amides and esters. ${ }^{[13-14]}$ Based on molecular modeling, the pentafluorophenyl unit appears to be bulky enough to prevent the pillar[5]arene moiety from escaping the thread of the rotaxane. The preparation of a [2]rotaxane with pentafluorophenyl ester stoppers was first tested starting from ethoxypillar[5]arene 1 and diacyl chloride 2 (Scheme 1).

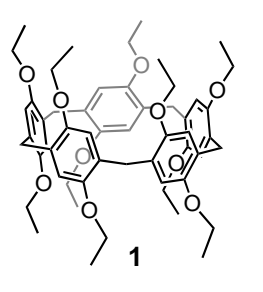

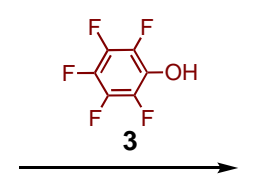

(i)
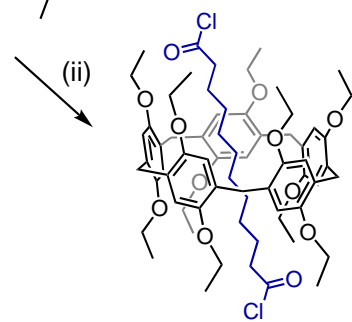

[1<2]
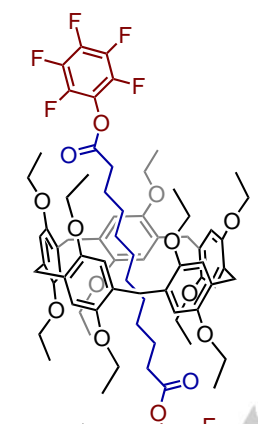

(iii)

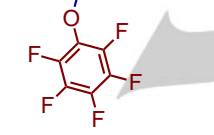

separated by column chromatography on silica gel. Rotaxane 4 was thus obtained pure in $75 \%$ yield and by-product 5 in $19 \%$ yield. Compound 5 was also prepared independently in $87 \%$ yield by treatment of diacyl chloride 2 with pentafluorophenol (3). Importantly rotaxane $\mathbf{4}$ and dumbbell $\mathbf{5}$ were perfectly stable on silica gel and partial hydrolysis of their ester functions was not observed as in the case of the corresponding 2,4-dinitrophenyl derivatives. The preparation of $\mathbf{4}$ was further improved by starting from a larger excess of 1 ( 3 equiv.). Under these conditions, the formation of the inclusion complex between 1 and 2 was more favorable and compound 4 was isolated in a nearly quantitative yield (98\%). The preparation of 4 was also achieved under mechanochemical solvent-free conditions (Scheme 1). ${ }^{[16-18]}$ In this particular case, the host-guest complex $[1 \subset 2]$ was prepared first and used in the solid state as received for the stoppering reaction. Specifically, a 2:1 mixture of 1 and 2 was dissolved in a minimum of $\mathrm{CHCl}_{3}{ }^{[16]}$ The resulting solution was cooled at $-15^{\circ} \mathrm{C}$ to favor the assembly of the desired inclusion complex and then evaporated to dryness without heating. The colorless solid thus obtained was transferred into a stainless-steel grinding vial containing pentafluorophenol (2.5 equiv.), $\mathrm{Et}_{3} \mathrm{~N}$ (2.5 equiv.) and four stainless steel balls. The resulting mixture was mixed in a Retsch MM400 mill at $30 \mathrm{~Hz}$ for $1 \mathrm{~h}$. Rotaxane 4 was thus obtained in $78 \%$ yield. The corresponding dumbbell (5) was also isolated as a by-product in $20 \%$ yield. To further improve the formation of inclusion complex $[1 \subset 2$ ], the same procedure was applied starting from a 3:1 mixture of $\mathbf{1}$ and $\mathbf{2}$. Under these conditions, 3 hours of mixing were necessary to complete the reaction. Rotaxane $\mathbf{4}$ was isolated in $94 \%$ yield and the corresponding unthreaded axle byproduct (5) could not be detected anymore. The outcome of the solvent-free stoppering reactions is therefore mainly dependent on the initial conditions used for the preparation of the neat samples of [1 $\subset 2$ ] but dissociation of the host-guest complex initially formed does not occur in the solid state during the mechanochemical treatment.

Rotaxane $\mathbf{4}$ was found stable for several months under normal laboratory conditions and could be stored without particular precaution. As anticipated, the pentafluorophenyl ester groups are large enough to prevent the pillar[5]arene moiety of $\mathbf{4}$ from escaping its axle. Compound $\mathbf{4}$ was effectively stable in solution and no dissociation could be evidenced even upon heating THF or $\mathrm{CHCl}_{3}$ solutions of $\mathbf{4}$ under reflux for several hours. The NMR and mass spectra recorded for $\mathbf{4}$ were in full agreement with the proposed structure (see ESI). The formation of rotaxane $\mathbf{4}$ was further evidenced by X-ray crystallography. Crystals suitable for $X$-ray crystal-structure analysis were obtained by slow diffusion of diethyl ether into a $\mathrm{CH}_{2} \mathrm{Cl}_{2}$ solution of compound 4 . As shown in Figure 1 , the $-\left(\mathrm{CH}_{2}\right)_{10}$ - chain of the axle component of rotaxane $\mathbf{4}$ does not adopt a fully extended form with all the successive methylene groups in an anti-conformation. Effectively, gauche conformations are almost exclusively observed for the decyl chain of $\mathbf{4}$ in the solid state. This peculiar conformation is most probably not related to the maximization of the intramolecular $\mathrm{C}-\mathrm{H} \cdots \pi$ interactions between the two components of the rotaxane. Effectively, in most of the X-ray crystal structures reported to date for analogous rotaxanes, ${ }^{[10-11]}$ the methylene units of the alkyl chains are typically in a fully extended all-anti conformation. In the particular case of $\mathbf{4}$, packing forces are likely responsible for the observed gauche conformations adopted by the decyl chain. The resulting 
contraction of the axle moiety allows most probably for optimized intermolecular contacts in the crystal lattice and the strain energy resulting from the adopted gauche conformations is thus compensated. This view is supported by the observation of intermolecular $\pi-\pi$ interactions of the electron-deficient pentafluorophenyl aromatic rings with electron-rich hydroquinone subunits of neighboring pillar[5]arenes leading to the formation of infinite supramolecular chains in the crystal lattice (Figure 1B).

(A)

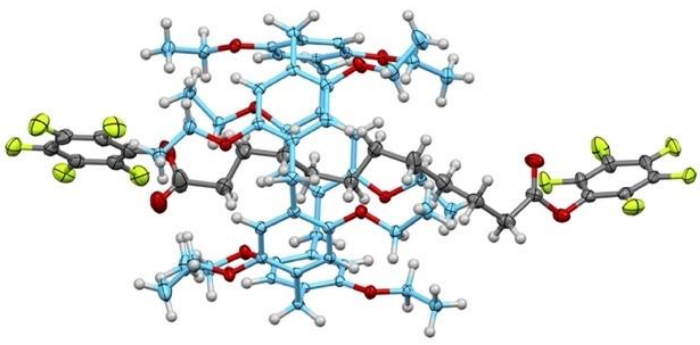

(B)

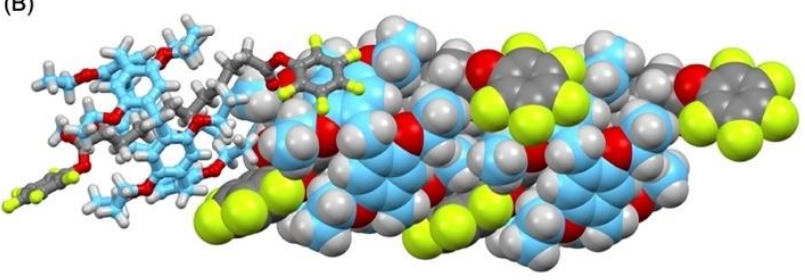

alkylamine reagents can be efficiently grafted onto the rotaxane scaffold by reaction with both pentafluorophenyl and 2,4dinitrophenyl ester building blocks. This strategy is however less efficient as soon as the nucleophilicity of the starting material is reduced by either steric hindrance in the case of secondary amines or electronic effects in the case of anilines. It can be noted that the reaction of 4 with amines $6 a$ and $6 c-g$ were also performed under mechanochemical solvent-free conditions. Whereas no stopper exchange reactions took place in the case of secondary amine $6 \mathrm{e}$ and anilines $6 \mathrm{f}-\mathrm{g}$, mixing 4 in the presence of an excess of the appropriate amine reagents $(6 \mathbf{a}$, 6c or 6d, 8 equiv.) in a Retsch MM400 mill at $30 \mathrm{~Hz}$ for 1.5 hours gave the corresponding rotaxanes $(7 \mathrm{a}, 7 \mathrm{c}$ and $7 \mathrm{~d})$. Under these optimized conditions, the isolated yields were similar to the ones obtained for the reactions performed in THF solutions. The use of a large excess of amine reagents (8 equiv.) was mandatory to achieve the stopper exchanges in good yields under mechanochemical conditions. This was not the case in solution where stochiometric amounts of the amine reagent are sufficient to complete the amide formation. For this reason, wet conditions are more convenient to achieve stopper exchange reactions from building block 4 and amine reagents.

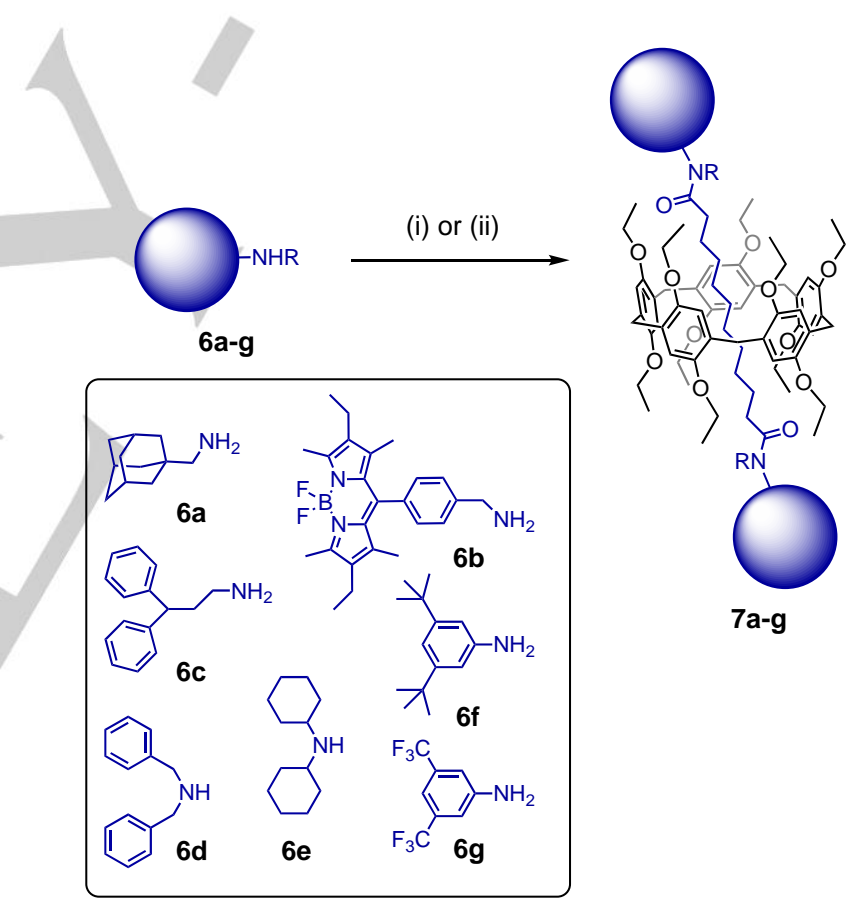

Scheme 2. Stopper exchange reactions with amine reagents. Reagents and conditions: (i) 4, THF (7a: quantitative, 7b: 96\%, 7c: $99 \%$, 7d: $26 \%, 7 e: 0 \%$, 7f: $62 \%, 7 g: 5 \%$ ); (ii) 4, mixing in a Retsch MM400 mill at $30 \mathrm{~Hz}$ for $1.5 \mathrm{~h} \mathrm{(7a:}$ 98\%, 7c: $92 \%, 7 d: 24 \%, 7 e-g: 0 \%)$.

The stopper exchange reactions of $\mathbf{4}$ with a series of phenol, alcohol and thiophenol reagents were also successfully achieved (Scheme 3). In our hands, related reactions starting from the rotaxane building block with 2,4-dinitrophenyl ester stoppers were at best low-yielding and decomposition of the starting material was typically faster than the formation of the desired products. The increased stability of the pentafluorophenyl ester subunits of $\mathbf{4}$ is therefore not only an advantage for purification purposes but it allows also to prepare 
rotaxanes with a larger structural diversity. Under optimized conditions, treatment of compound $\mathbf{4}$ with an excess of phenol 8a (4 equiv.) in the presence of 1,8-diazabicyclo(5.4.0)undec-7ene (DBU, 2.05 equiv.) in THF as a base provided rotaxane 9a in $57 \%$ yield. Applying these conditions to the other phenol or alcohol reagents gave however unsatisfactory results and the desired rotaxanes were generally thus obtained in low to moderate yields. Side products resulting from the partial hydrolysis of the rotaxanes were also typically formed under these conditions. This prompted us to test other organocatalysts for the transesterification reactions. The best results were obtained with 1,4,7-triazabicyclo[4.4.0]dec-5-ene (TBD). ${ }^{[14]}$ Reaction of $\mathbf{4}$ with the phenol or alcohol starting materials (8a-h, 4 equiv.) and TBD (2.05 equiv.) in THF at room temperature gave the corresponding rotaxanes $9 \mathrm{a}-\mathrm{h}$ without formation of byproducts. The use of a large excess of $\mathbf{8 a}-\mathbf{h}$ was mandatory for a complete conversion. This observation suggests that an equilibrium is reached in solution and the excess of $\mathbf{8 a}-\mathbf{h}$ is necessary to fully drive the equilibrium towards the formation of the desired products. Rotaxanes $9 \mathbf{a}-\mathbf{h}$ were thus prepared in good yields. The only exception was $9 \mathrm{~b}$. In this particular case the bis(trifluromethyl)phenyl ester is also an activated ester ${ }^{[12 f]}$ and the use of $\mathbf{4}$ equiv. of $\mathbf{8 b}$ was certainly not sufficient to fully displace the equilibrium towards the complete formation of $\mathbf{9 b}$. The conditions used for the preparation of rotaxane diesters $9 \mathrm{a}$ $\mathbf{h}$ were also successful for the preparation of $9 \mathbf{i}$ starting from 4 and thiophenol $\mathbf{8 i}$. Rotaxane $\mathbf{9 i}$ with two thiophenyl ester stoppers was thus obtained in $71 \%$ yield.
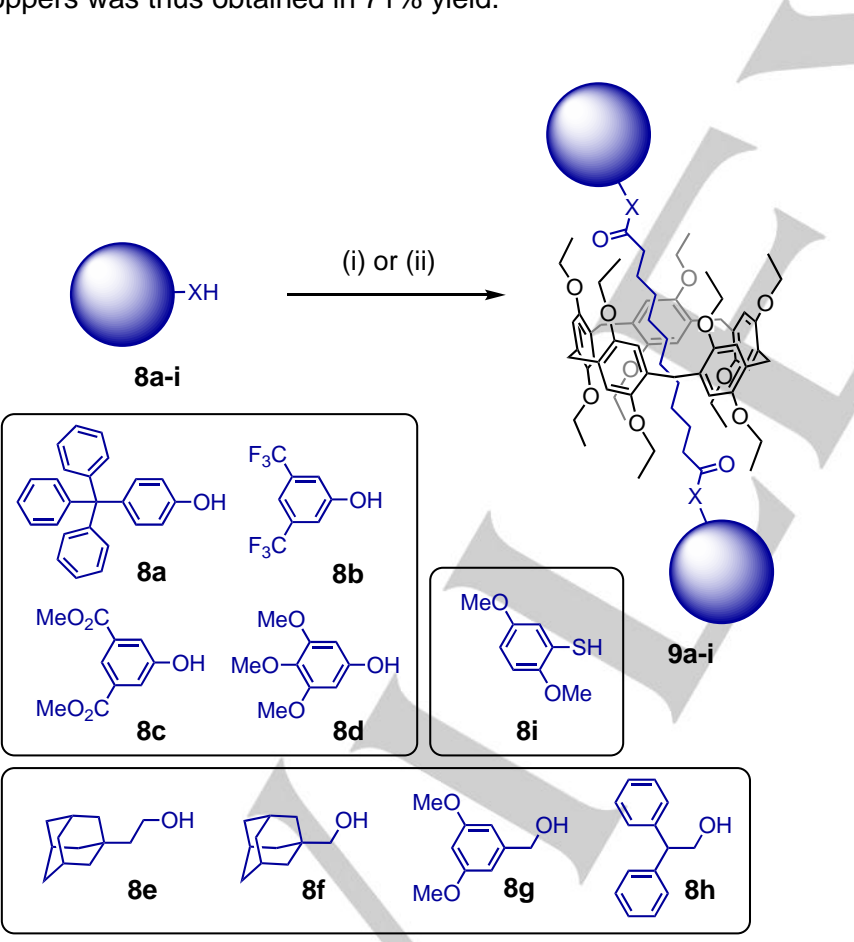

Scheme 3. Stopper exchange reactions with phenol, alcohol and thiophenol reagents. Reagents and conditions: (i) 4, TBD, THF (9a: $86 \%$, 9b: 19\%, 9c: $79 \%$, 9d: $97 \%$, 9e: $86 \%$, 9f: $63 \%$, 9g: $80 \%$, 9h: $81 \%$, 9i: $71 \%$ ); (ii) 4, TBD, mixing in a Retsch MM400 mill at $30 \mathrm{~Hz}$ for 2 h (9a: $82 \%, 9 b: 88 \%, 9 c: 92 \%$, 9d: $78 \%$, 9e: $86 \%$, 9f: $88 \%$, 9g: $87 \%$, 9h: $87 \%$, 9i: $97 \%$ ).

Compounds 9a-i were also prepared under mechanochemical solvent-free conditions (Scheme 3). A mixture of rotaxane 4 , the appropriate nucleophile (8a-i, 2.3 equiv.) and TBD (2.3 equiv.) was mixed in a Retsch MM400 mill at $30 \mathrm{~Hz}$ for $2 \mathrm{~h}$. Rotaxanes 9a-i were thus obtained in $78-97 \%$ yields. The transesterification reactions were clearly more efficient under the solvent-free conditions. Moreover, only a slight excess of 8 a-i was necessary to complete the reactions thus making the purification of the resulting rotaxanes easier.

All the rotaxanes were fully characterized by NMR and IR spectroscopies and mass spectrometry. Their purity was confirmed by elemental analysis data. Crystals suitable for X-ray structure analysis were obtained for rotaxanes $9 \mathrm{c}$ and $9 \mathrm{~d}$. The structures are depicted in Figure 2.

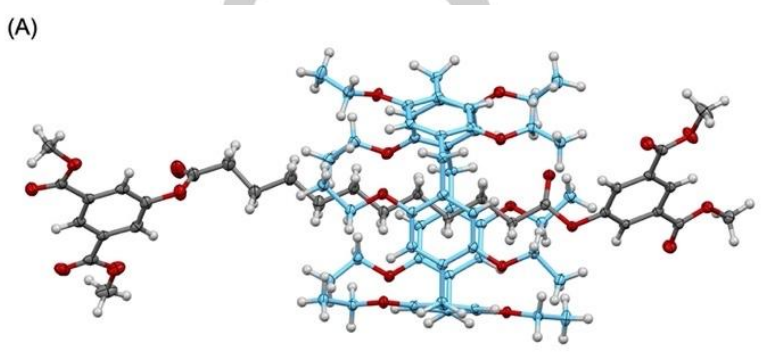

(B)
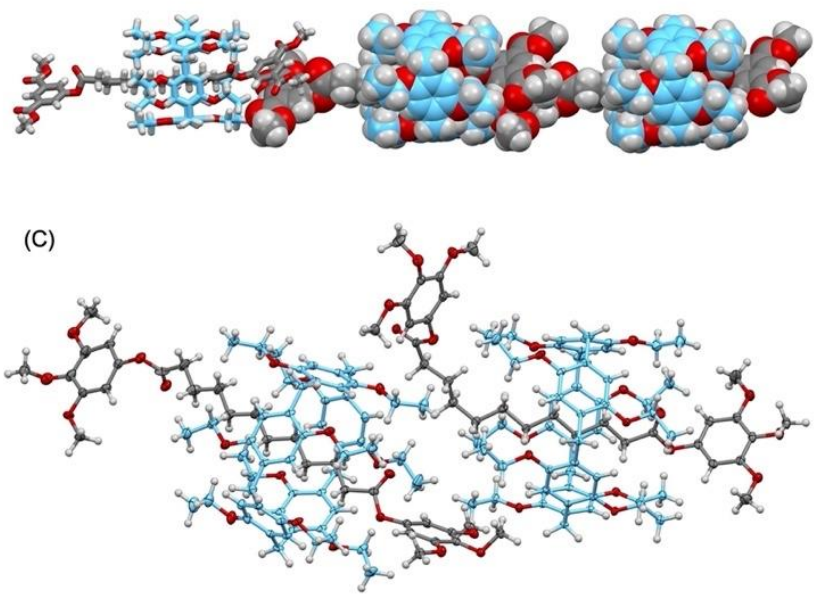

Figure 2. (A) ORTEP plot of the structure of $9 \mathrm{c}$ (the two co-crystallized $\mathrm{Et}_{2} \mathrm{O}$ molecules are omitted for clarity, thermal ellipsoids are shown at $30 \%$ probability level). (B) Stacking within the 9c lattice highlighting the intermolecular $\pi-\pi$ interactions between the stoppers of neighbouring rotaxanes. (C) ORTEP plot of the two conformers of 9d present in the crystal lattice (thermal ellipsoids are shown at $30 \%$ probability level). Colour code: $\mathrm{H}$ : white, O: red, C: grey for the dumbbell and pale blue for the pillar[5]arene moiety.

Both compounds are chiral owing to their $D_{5}$-symmetry imposed by the pillar[5]arene subunits. Both $9 \mathbf{c}$ and $9 \mathbf{d}$ crystallized as racemates. Close inspection of the packing in the crystal lattice of $9 c$ along the crystallographic a axis revealed alternated layers of both enantiomers. Within these layers, the rotaxanes are organized in infinite supramolecular chains through the establishment of $\pi-\pi$ interactions between the stoppers of neighboring molecules (Figure 2B). As a result, this supramolecular organization does not generate specific packing constrains as observed in the case of $\mathbf{4}$. Therefore, the decyl chain of the axle component of $9 \mathrm{c}$ is not compressed by the packing forces and adopts a fully extended all-anti conformation in the solid state. In the case of rotaxane $9 d$, the supramolecular organization is more complex due to the existence of two 
conformers within the crystal lattice (Figure 2C). For the first one, the decyl chains adopts a linear all anti conformation. In contrast, the second one is twisted due to the gauche conformation adopted by two successive methylene subunits located outside the cavity of the pillar[5]arene moiety. In the case of $9 d$, close inspections of the crystal lattice revealed no particular $\pi-\pi$ interactions between neighboring molecules and the packing is mainly dominated by Van der Waals forces.

Preparation of fullerene-containing photoactive rotaxanes. The preparation of a wide range of rotaxanes was successively achieved by stopper exchange reactions from key precursor 4 . To further evaluate the potential of this new methodology, it was decided to prepare building block $\mathbf{1 1}$ starting from fulleropillar[5]arene $\mathbf{1 0}$ in order to prepare a photoactive MIM by the grafting of complementary photoactive stoppers to the fullerorotaxane scaffold (Scheme 4). Compound 10 was prepared as previously described. ${ }^{[9]}$ Owing to the moderate solubility of $\mathbf{1 0}$ in $\mathrm{CHCl}_{3}$, the reaction conditions optimized for the preparation of compound $\mathbf{4}$ had to be adapted. In order to favor the incorporation of $\mathbf{1 0}$ in host-guest complexes, an excess of diacyl chloride 2 ( 3 equiv.) was used in this case. Treatment of the 3:1 mixture of $\mathbf{2}$ and $\mathbf{1 0}$ dissolved in a minimum of $\mathrm{CHCl}_{3}$ with pentafluorophenol (3) in the presence of $\mathrm{Et}_{3} \mathrm{~N}$ at $-15^{\circ} \mathrm{C}$ afforded 11. After work-up and purification, rotaxane 11 was obtained in $82 \%$ yield. The ${ }^{1} \mathrm{H}$ NMR spectrum of $\mathbf{1 1}$ showing the diagnostic signals of both subunits was fully consistent with the proposed structure. Moreover, the dramatic shielding observed for the signals arising from the methylene groups of the axle moiety provides definitive evidence for the interlocked structure of 11. The up-field shifted chemical shifts of these signals results actually from their location within the cavity of the macrocycle and is due to the ring current effect of the fulleropillar[5]arene hydroxyquinone subunits. The MALDI-TOF mass spectrum of $\mathbf{1 1}$ showing the expected molecular ion peak at $\mathrm{m} / \mathrm{z}=2170.65\left([\mathrm{M}]^{+}\right.$ calcd for $\mathrm{C}_{139} \mathrm{H}_{88} \mathrm{~F}_{10} \mathrm{O}_{14}: 2170.60$ ) as the base peak fully confirmed the structure of this compound. The reaction conditions developed for the preparation of diamide [2]rotaxanes from 4 were then applied to $\mathbf{1 1}$. Treatment of $\mathbf{1 1}$ with primary amine $\mathbf{6 a}$ in THF provided rotaxane $12 \mathbf{a}$ in $67 \%$ yield. Finally, incorporation of photoactive boron-dipyrromethene (Bodipy) stoppers was achieved by reaction of $\mathbf{1 1}$ with $\mathbf{6 b}$. For solubility reasons, the stopper exchange reaction was carried out in a 2:1 $\mathrm{THF} / \mathrm{CHCl}_{3}$ mixture. Photoactive MIM 12b was thus obtained in $71 \%$ yield. Rotaxanes 12a-b were characterized by a combination of several analytical techniques. In both cases, the expected molecular ion peak was observed in their MALDI-TOF mass spectra. The NMR data were also fully consistent with the proposed structures. Compound $\mathbf{1 2 b}$ combines complementary chromophore subunits, namely a fullerene moiety grafted onto the macrocyclic unit and two Bodipy subunits acting as stoppers. ${ }^{[19]}$ The ground state absorption spectra of rotaxane 12b and model compounds $6 \mathbf{b}, \mathbf{7 b}$ and $12 \mathbf{a}$ recorded in $\mathrm{CH}_{2} \mathrm{Cl}_{2}$ are depicted in Figure 3. The absorption spectrum of model Bodipy compounds $\mathbf{6 b}$ and $\mathbf{7 b}$ are dominated by an intense band centered at 524 and $526 \mathrm{~nm}$, respectively. This band is attributed to the lowest energy spin-allowed $\pi-\pi^{*}$ transition of the Bodipy chromophore. ${ }^{[20]}$ The typical higher energy $\pi-\pi^{*}$ transitions of the bodipy moieties ${ }^{[20]}$ are also observed in the 280-380 nm region for both compounds. Comparison of the molar extinction coefficients $(\varepsilon)$ of $\mathbf{6 b}$ and $\mathbf{7 b}$ are fully consistent with the presence of two Bodipy subunits in the rotaxane and the differences observed for $\mathbf{6 b}$ and $\mathbf{7 b}$ between 270 and $340 \mathrm{~nm}$ are due to transitions centered on the pillar[5]arene moiety of $\mathbf{7 b}$ which are also contributing to the overall absorption in the UV region. On the other hand, the absorption spectrum of model rotaxane 12a displays the characteristic features of methanofullerene derivatives ${ }^{[21]}$ with a very intense band in the UV $\left(259 \mathrm{~nm}, \varepsilon=113800 \mathrm{M}^{-1} \mathrm{~cm}^{-1}\right)$ and a much weaker and broad absorption in the all visible region. The very weak band observed at $702 \mathrm{~nm}\left(\varepsilon=320 \mathrm{M}^{-1} \mathrm{~cm}^{-1}\right)$ is attributable to the lowest singlet transition. ${ }^{[21]}$ The absorption spectrum of multicomponent fullerene-Bodipy system $\mathbf{1 2 b}$ shows the characteristic features of both chromophores. Comparison with the absorption spectra of the corresponding model compounds $\mathbf{7 b}$ and $12 a$ revealed only very minor differences and groundstate electronic interactions between the Bodipy stoppers and the central $\mathrm{C}_{60}$ subunit are negligible.

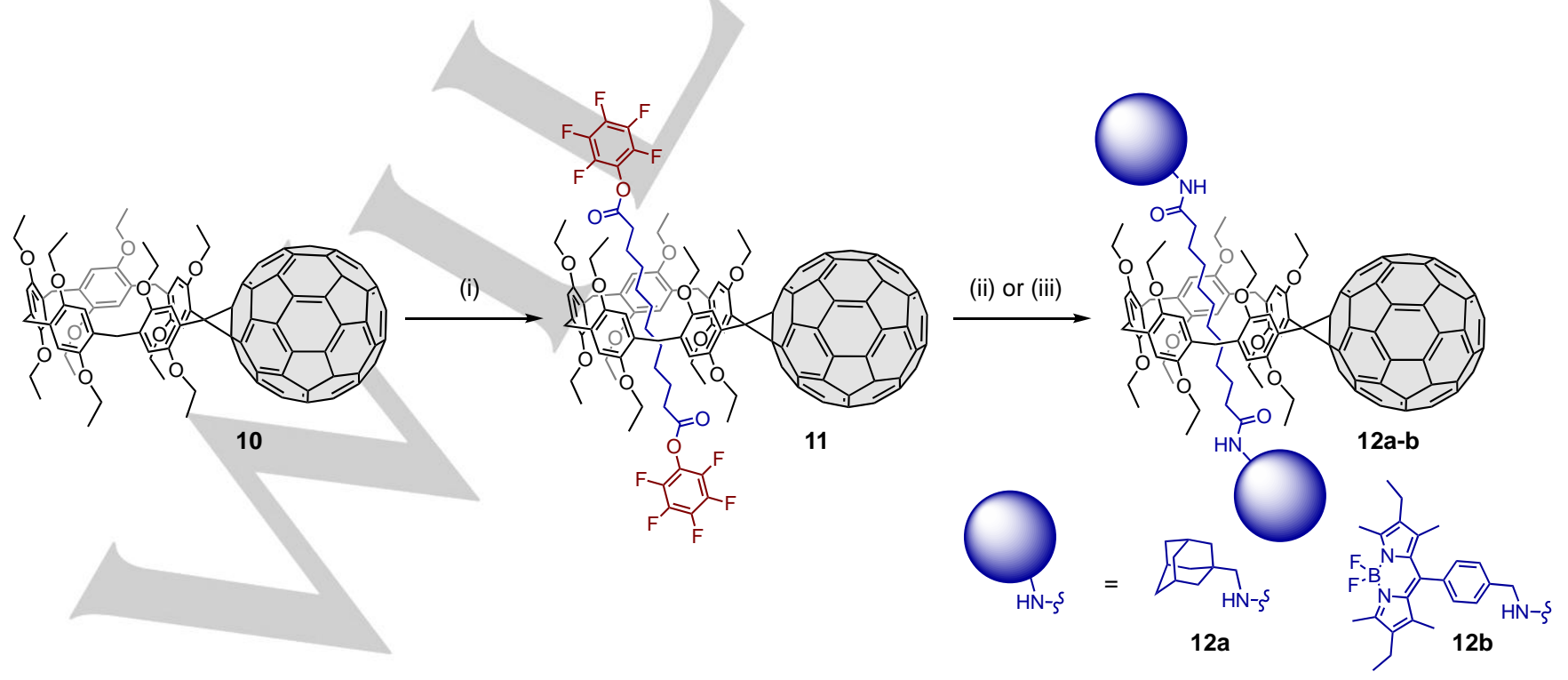


Scheme 4. Preparation of fullerene-containing rotaxanes. Reagents and conditions: (i) 2 , 3, $\mathrm{Et}_{3} \mathrm{~N}, \mathrm{CHCl}_{3},-15^{\circ} \mathrm{C}(82 \%)$; (ii) $6 \mathbf{a}, \mathrm{THF}(12 \mathrm{a}: 67 \%)$; (iii) $6 \mathrm{~b}, \mathrm{THF} / \mathrm{CHCl}{ }_{3}$ (12b: $71 \%)$.
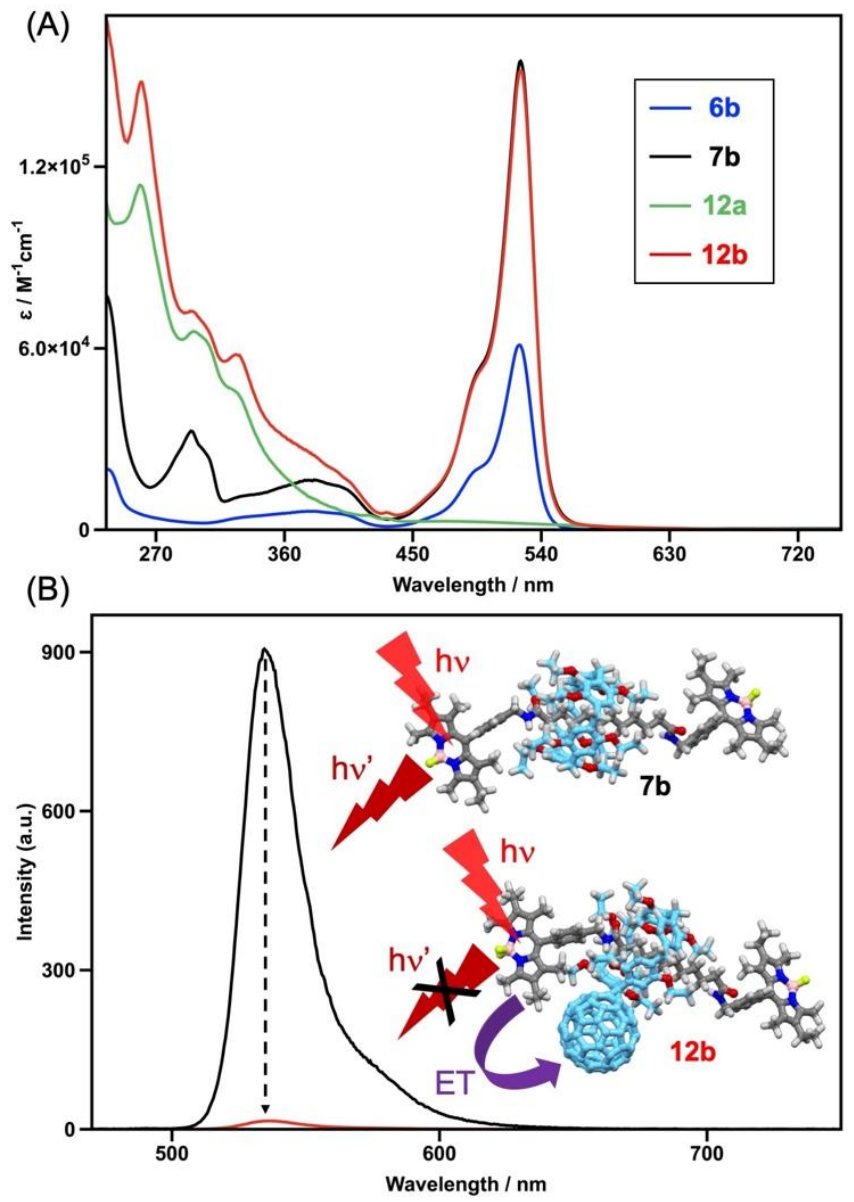

Figure 3. (A) Absorption spectra of $\mathbf{6 b}$ (blue), $\mathbf{7 b}$ (black), 12a (green) and 12b (red) recorded in $\mathrm{CH}_{2} \mathrm{Cl}_{2}$ at $25^{\circ} \mathrm{C}$. (B) Emission spectra of isoabsorbing solutions of compounds $7 \mathbf{b}$ (black) and $12 \mathrm{~b}$ (red) in $\mathrm{CH}_{2} \mathrm{Cl}_{2}$ at $25^{\circ} \mathrm{C}\left(\lambda_{\text {exc }}=490\right.$ $\mathrm{nm}$ ) and schematic representation of the photoexcitation of the Bodipy moiety followed by a strong emission in the case of $\mathbf{7 b}$ and by energy transfer (ET) in the case of $\mathbf{1 2 b}$.

Emission spectra were also recorded for compounds $\mathbf{6 b}, \mathbf{7 b}$ and 12a-b in $\mathrm{CH}_{2} \mathrm{Cl}_{2}$ solutions at room temperature. Under our experimental conditions, the extremely weak fullerene emission $\left(\phi_{\mathrm{em}}<0.0001\right)^{[21]}$ could not be detected for compound 12a. In contrast, model compounds $\mathbf{6 b}$ and $\mathbf{7 b}$ exhibit the typical strong emission of Bodipy dyes at $535 \mathrm{~nm}\left(\lambda_{\mathrm{exc}}=490 \mathrm{~nm}\right)$. As shown in Figure $3 \mathrm{~B}$, the bodipy fluorescence is still detected for compound 12b upon excitation at $490 \mathrm{~nm}$ where most of the light is absorbed by the Bodipy. However, comparison of the emission intensity of isoabsorbing solutions of $\mathbf{7 b}$ and $\mathbf{1 2 b}$ revealed a dramatic quenching (ca. $96 \%$ ) of the Bodipy emission by the fullerene moiety in $\mathbf{1 2 b}$ despite the absence of covalent linkage between these chromophores (Figure 3B). The bodipy dyes used as stoppers in $\mathbf{1 2 b}$ are not particularly strong electron donors neither in their ground nor excited states. ${ }^{[22]}$ The Bodipy singlet excited state in $\mathbf{1 2 b}$ is most likely quenched by an efficient energy transfer to populate the first fullerene singlet

excited state. Whereas it was not possible to fully confirm the energy transfer mechanism by monitoring the weak fullerene emission with our experimental setup, the low electron donating ability of the Bodipy stoppers in $\mathbf{1 2 b}$ was supported by DFT calculations (see ESI for the details). The highest occupied molecular orbital (HOMO) is indeed located on hydroquinone subunits of the pillar[5]arene subunit of $12 \mathrm{~b}$ and not on the Bodipy stoppers. Only the HOMO-1 and HOMO-2 were found on the Bodipy subunits (Figure 4). As expected, the lowest occupied molecular orbital (LUMO) is located on the fullerene moiety of $\mathbf{1 2 b}$.

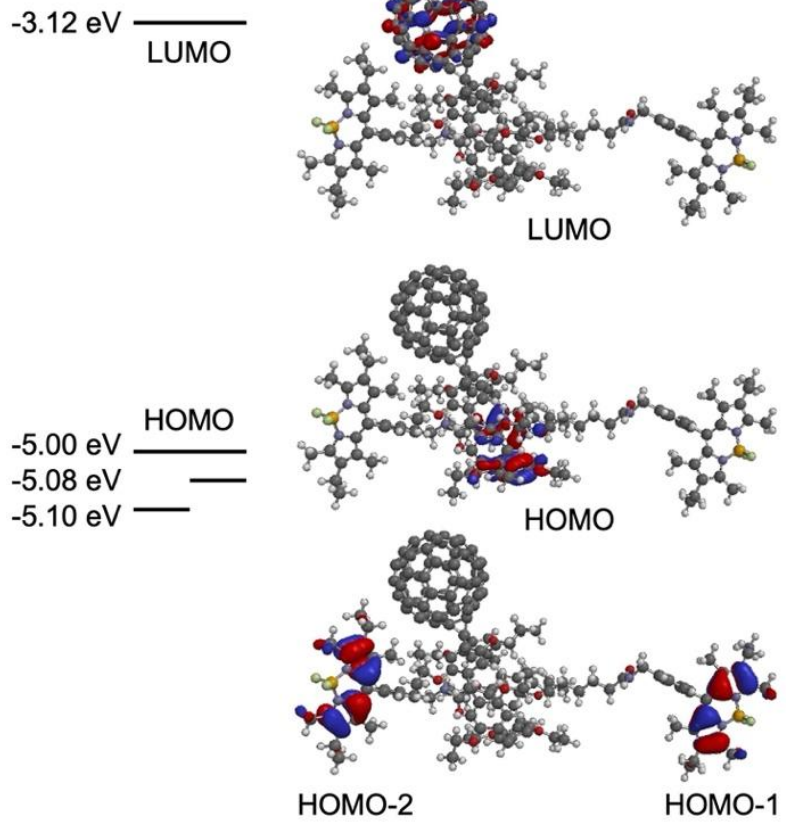

Figure 4. Frontier molecular orbitals calculated at the B3LYP/6-31G* level for compound $12 \mathrm{~b}$. In the calculated structure, the boron atoms of the two stoppers are separated by a distance of $32.18 \AA$.

Owing to possible shuttling motions of the pillar[5]arene moiety along the axle of rotaxane $\mathbf{1 2 b}$ and to the overall conformational flexibility of the system, the Bodipy-fullerene distance is fluctuating over a large range. Based on the theoretical calculations, when the fullerenopillar[5]arene is close to one Bodipy stopper in 12b (Bodipy-fullerene distance of ca. $4 \AA$ ), the distance to the second Bodipy unit is quite large (ca. $22 \AA$ ). The though space quenching of the Bodipy emission is very efficient and apparently insensitive to dynamic fluctuations of the Bodipyfullerene distance in 12b. This is also in favor for a Bodipy to fullerene singlet-singlet energy transfer mechanism upon photoexcitation of the Bodipy subunits.

\section{Conclusion}


Rotaxane 4 incorporating pentafluorophenol ester stoppers has been prepared in a nearly quantitative yield. This building block is easily affordable on a multi-gram scale. It is also perfectly stable under standard laboratory conditions thus allowing storage for several months before further transformation. Stopper exchange reactions have been carried out from $\mathbf{4}$ and various nucleophiles to generate a wide range of pillar[5]arenecontaining rotaxanes in high yields. The pentafluorophenyl ester activation method has been also applied to the construction of more sophisticated [2]rotaxanes incorporating a fullerenefunctionalized pillar[5]arene moiety. This allowed us to easily prepare a photoactive MIM in which through space intercomponent photoinduced events have been evidenced. The versatility of this new methodology and its application for the easy construction of sophisticated rotaxanes are clear advantages for future developments in the field as the preparation of new molecular machines, advanced materials or bioactive compounds based on MIMs will not be limited by their synthesis anymore.

\section{Experimental Section}

\section{Synthesis}

The preparation and characterization of all the new compounds are described in the Supporting Information. Compounds $\mathbf{1}^{[23]}, \mathbf{6} \mathrm{b}^{[24]}$ and $\mathbf{1 0 ^ { [ 9 ] }}$ were prepared according to previously reported procedures.

\section{X-ray crystal structures}

The crystallographic data and the refinement parameters are reported in the Supporting Information for all the compounds. The X-ray crystal structures have been deposited at the Cambridge Structural Database (CCDC deposition numbers: 2058646 for 4, 2058647 for 9c, and 2059867 for 9d). These data are provided free of charge by the joint Cambridge Crystallographic Data Centre and Fachinformationszentrum Karlsruhe Access Structures (https://www.ccdc.cam.ac.uk/structures).

\section{Acknowledgements}

Financial support by the ANR (projects FastGiant ANR-17CE07-0012-01 and Pillar ANR-19-CE06-0032), the International Center for Frontier Research in Chemistry and the LabEx "Chimie des Systèmes Complexes" is gratefully acknowledged. We thank L. Karmazin and C. Bailly for the X-ray crystal structure resolution, E. Wasielewski for high-field NMR measurements and J.M. Strub for the mass spectra. We further thank A. N. Kursunlu for the initial preparation of starting material $\mathbf{6 b}$.

Keywords: Stopper exchange - Pillar[5]arene • Rotaxanes • Supramolecular chemistry $\cdot$ Photoactive systems

[1] a) J.-P. Sauvage, Angew. Chem. Int. Ed. 2017, 56, 11080-11093 Angew. Chem. 2017, 129, 11228-11242; b) J. F. Stoddart, Angew. Chem. Int. Ed. 2017, 56, 11228-11242; Angew. Chem. 2017, 129, 11244-11277; c) B. L. Feringa, Angew. Chem. Int. Ed. 2017, 56, 1106011078; Angew. Chem. 2017, 129, 11228-11242.
[2] a) V. Balzani, A. Credi, M. Venturi, Molecular Devices and Machines A Journey into the Nano World, Wiley-VCH, 2003; b) J.-P. Sauvage, C. Dietrich-Buchecker (Eds.), Molecular Catenanes, Rotaxanes and Knots - A Journey Through the World of Molecular Topology, J. Wiley and Sons, 2008; c) E. R. Kay, D. A. Leigh, Angew. Chem. Int. Ed. 2015, 54, 10080-10088; Angew. Chem. 2015, 127, 10218-10226; d) C. J. Bruns, J. F. Stoddart, The Nature of the Mechanical Bond - From Molecules to Machines, J. Wiley \& Sons, 2016; e) S. Erbas-Cakmak, D. A. Leigh, C. T. McTernan, A. L. Nussbaumer, Chem. Rev. 2015, 115, 10081-10206; f) D. A. Leigh, Angew. Chem. Int. Ed. 2016, 55, 14506-14508; Angew. Chem. 2016, 128, 14722-14724; g) S.-J. Rao, Q. Zhang, J. Mei, X.-H. Ye, C. Gao, Q.-C. Wang, D.-H. Qu, H. Tian, Chem. Sci. 2017, 8, 67776783; h) S.-J. Rao, K. Nakazono, X. Liang, K. Nakajima, T. Takata, Chem. Commun. 2019, 55, 5231-5234; i) Q. Zhang, S.-R. Rao, T. Xie, X. Li, T.-Y. Xu, D.-W/ Li, D.-H. Qu, Y.-T. Long, H. Tian, Chem 2018, 4, 2670-2684.

[3] a) J.-C. Chambron, V. Heitz, J.-P. Sauvage, J. Chem. Soc., Chem. Commun. 1992, 1131-1133; b) J.-C. Chambron, A. Harriman, V. Heitz, J.-P. Sauvage, J. Am. Chem. Soc. 1993, 115, 6109-6114; c) J.-C. Chambron, A. Harriman, V. Heitz, J.-P. Sauvage, J. Am. Chem. Soc. 1993, 115, 7419-7425; d) J.-C. Chambron, V. Heitz, J.-P. Sauvage, J. Am. Chem. Soc. 1993, 115, 12378-12384.; e) M. Linke, J.-C. Chambron, V. Heitz, J.-P. Sauvage, J. Am. Chem. Soc. 1997, 119, 11329-11330; f) M.-J. Blanco, J.-C. Chambron, V. Heitz, J.-P. Sauvage, Org. Lett. 2000, 2, 3051-3054; g) M. Linke, J.-C. Chambron, V. Heitz, J.-P. Sauvage, S. Encinas, F. Bargelletti, L. Flamigni, J. Am. Chem. Soc. 2000, 122, 11834-11844; h) M. Andersson, M. Linke, J.-C. Chambron, J. Davidsson, V. Heitz, L. Hammarström, J.-P. Sauvage, J. Am. Chem. Soc. 2002, 124, 4347-4362.

[4] For reviews, see: a) A. Harriman, J.-P. Sauvage, Chem. Soc. Rev 1996, 25, 41-48; b) M.-J. Blanco, M. C. Jiménez, J.-C. Chambron, V. Heitz, M. Linke, J.-P. Sauvage, Chem. Soc. Rev. 1999, 28, 293-305; c) J. A. Faiz, V. Heitz, J.-P. Sauvage, Chem. Soc. Rev. 2009, 38, 422-442.

[5] a) F. Diederich, C. Dietrich-Buchecker, J.-F. Nierengarten, J.-P. Sauvage, J. Chem. Soc., Chem. Commun. 1995, 781-782; b) N. Armaroli, F. Diederich, C. O. Dietrich-Buchecker, L. Flamigni, G. Marconi, J.-F. Nierengarten, J.-P. Sauvage, Chem. Eur. J. 1998, 4, 406-416; c) J. D. Megiatto Jr., R. Spencer, D. I. Schuster, Org. Lett. 2009, 11, 4152-4155; d) J. D. Megiatto Jr., K. Li, D. I. Schuster, A. Palkar, M. A. Herranz, L. Echegoyen, S. Abwandner, G. de Miguel, D. M. Guldi, J. Phys. Chem. B 2010, 114, 14408-14419; e) J. D. Megiatto Jr., D. I. Schuster, S. Abwandner, G. de Miguel, D. M. Guldi, J. Am. Chem. Soc. 2010, 132, 3847-3861.

[6] For a review, see: J. D. Megiatto Jr., D. I. Schuster, D. M. Guldi, Chem. Soc. Rev. 2020, 49, 8-20.

[7] For selected examples, see: a) S. Saha, A. H. Flood, J. F. Stoddart, S Impellizzeri, S. Silvi, M. Ventura, A. Credi, J. Am. Chem. Soc. 2007, 129, 12159-12171; b) T. Ogoshi, D. Yamafuji, T.-a. Yamagishi, A. M. Brouwer, Chem. Commun. 2013, 49, 5468-5470; c) T. A. Barendt, I. Rasovic, M. A. Lebedeva, G. A. Farrow, A. Auty, D. Chekulaev, I. V. Sazanovich, J. A. Weinstein, K. Porfyrakis, P. D. Beer, J. Am. Chem. Soc. 2018, 140, 1924-1936; d) Y. Xu, R. Kaur, B. Wang, M. B. Minameyer, S. Gsänger, B. Meyer, T. Drewello, D. M. Guldi, M. von Delius, J. Am. Chem. Soc. 2018, 140, 13413-13420; e) M. Wolf, A. Ogawa, M. Bechtold, M. Vonesch, J. A. Wytko, K. Oohara, S. Campidelli, T. Hayashi, D. M. Guldi, J. Weiss, Chem. Sci. 2019, 10, 3846-3853; f) S. Yang, C.-AX. Zhao, S. Crespi, X. Li, Q. Zhang, Z.-H. Zhang, J. Mei, H. Tian, D.-H. Qu, Chem 2021, DOI: 10.1016/j.chempr.2021.02.019.

[8] B. Delavaux-Nicot, H. Ben Aziza, I. Nierengarten, T. M. N. Trinh, E Meichsner, M. Chessé, M. Holler, R. Abidi, E. Maisonhaute, J.-F. Nierengarten, Chem. Eur. J. 2018, 24, 133-140.

[9] E. Meichsner, I. Nierengarten, M. Holler, M. Chessé, J-F. Nierengarten, Helv. Chim. Acta 2018, 101, e1800059.

[10] I. Nierengarten, E. Meichsner, M. Holler, P. M. Pieper, R. Deschenaux, B. Delavaux-Nicot, J.-F. Nierengarten, Chem. Eur. J. 2018, 24, 169-177.

[11] I. Nierengarten, J.-F. Nierengarten, ChemistryOpen 2020, 9, 393-400.

[12] The post-modification of pre-constructed [2]rotaxanes by stopper exchange reactions has been rarely used and only a few examples 
have been reported so far, see: a) S. J. Rowan, J. F. Stoddart, J. Am. Chem. Soc. 2000, 122, 164-165; b) S. J. Rowan, S. J. Cantrill, J. F Stoddart, A. J. P. White, D. J. Williams, Org. Lett. 2000, 2, 759-762; c) R. J. Bordoli, S. M. Goldup, J. Am. Chem. Soc. 2014, 136, 4817-4820 d) D. W. Zehnder II, D. B. Smithrud, Org. Lett. 2001, 3, 2485-2487; e) N. Kihara, S. Motoda, T. Yokozawa, T. Takata, Org. Lett. 2005, 7, 1199 1202 ; f) J. S. Hannam, S. M. Lacy, D. A. Leigh, C. G. Saiz, A. M. Z Slawin, S. G. Stitchell, Angew. Chem. Int. Ed. 2004, 43, 3260-3264 Angew. Chem. 2004, 116, 3322-3326; g) S. C. Rajappan, D. R McCarthy, J. P. Campbell, J. B. Ferrel, M. Sharafi, O. Ambrozaite, J. Li, S. T. Schneebeli, Angew. Chem. Int. Ed. 2020, 59, 16668-16674 Angew. Chem. 2020, 132, 16811-16817; h) For a recent review on post-synthetic modification of interlocked molecules, see: P. Waelès, M. Gauthier, F. Coutrot, Angew. Chem. Int. Ed. doi.org/10.1002/anie.202007496; Angew. Chem. doi.org/10.1002/ange.202007596.

[13] S.-Y. Han, Y.-A. Kim, Tetrahedron 2004, 2447-2467.

[14] S. R. Samanta, R. Cai, V. Percec, Polym. Chem. 2015, 6, 3259-3270.

[15] R. Milev, A. Lopez-Pacheco, I. Nierengarten, T. M. N. Trinh, M. Holler, R. Deschenaux, J.-F. Nierengarten, Eur. J. Org. Chem. 2015, 479-485.

[16] M. Holler, T. Stoerkler, A. Louis, F. Fischer, J.-F. Nierengarten, Eur. J. Org. Chem. 2019, 3401-3405.

[17] For other examples of mechanochemical synthesis of MIMs, see: a) A. Orita, J. Okano, Y. Tawa, L. Jiang, J. Otera, Angew. Chem. Int. Ed. 2004, 43, 3724-3728; Angew. Chem. 2004, 116, 3810-3814; b) S. Y Hsueh, K. W. Cheng, C.-C. Lai, S.-H. Chiu, Angew. Chem. Int. Ed. 2008, 47, 4436-4439; Angew. Chem. 2008, 47, 4508-4511; c) C.-C Hsu, N. C. Chen, C.-C. Lai, Y.-H. Liu, S.-M. Peng, S.-H. Shiu, Angew. Chem. Int. Ed. 2008, 47, 7475-7478; Angew. Chem. 2008, 47, 7585 7588; d) C.-C. Hsu, C.-C. Lai, S.-H. Chiu, Tetrahedron 2009, 65, 2824 2829; e) P.-N. Chen, C.-C. Lai, S.-H. Chiu, Org. Lett. 2011, 13, 4660 4663; f) K.-D. Wu, Y.-H. Lin, C.-C. Lai, S.-H. Chiu, Org. Lett. 2014, 16 1068-1071; g) H.-G. Li, G.-W. Wang, J. Org. Chem. 2017, 82, 63416348.

[18] For reviews on supramolecular concepts in mechanochemistry, see: a) T. Friščić, Chem. Soc. Rev. 2012, 41, 3493-3510; b) H.-G. Li, L. Li, G.W. Wang, Current Org. Chem. 2018, 22, 923-929; c) A. Inthasot, T.-Y. Tai, S.-H. Chiu, J. Chin. Chem. Soc. 2019, 66, 134-145.

[19] For selected examples of covalent photoactive molecular devices combining $\mathrm{C}_{60}$ with Bodipy dyes, see: a) R. Ziessel, B. D. Allen, D. B. Rewinska, A. Harriman, Chem. Eur. J. 2009, 15, 7382-7393; b) J.-Y. Liu, M. E. El-Khouly, S. Fukuzumi, D. K. P. Ng, Chem. Asian J. 2010, 6, 174-179; c) J. lehl, J.-F. Nierengarten, A. Harriman, T. Bura, R. Ziessel, J. Am. Chem. Soc. 2012, 134, 988-998; d) V. Bandi, H. B. Gobeze, P. A. Karr, F. D'Souza, J. Phys. Chem. C 2014, 118, 18969-18982; e) C. B. KC, G. N. Lim, V. N. Nesterov, P. A. Karr, F. D'Souza, Chem. Eur. J. 2014, 20, 17100-17112.

[20] A. Loudet, K. Burgess, Chem. Rev. 2007, 107, 4891-4932.

[21] a) N. Armaroli, G. Accorsi, D. Felder, J.-F. Nierengarten, Chem. Eur. J. 2002, 8, 2314-2323; b) J.-F. Eckert, J.-F. Nicoud, J.-F. Nierengarten, S.-G. Liu, L. Echegoyen, F. Barigelletti, N. Armaroli, L. Ouali, V. Krasnikov, G. Hadziioannou, J. Am. Chem. Soc. 2000, 122, 7467-7479.

[22] T. Lazarides, G. Charalambidis, A. Vuillamy, M. Réglier, E. Klontzas, G. Froudakis, S. Kuhri, D. M. Guldi, A. G. Coutsolelos, Inorg. Chem. 2011, 50, 8926-8936.

[23] a) T. Ogoshi, K. Kitajima, T. Aoki, S. Fujinami, T.-a. Yamagishi, Y. Nakamoto, J. Org. Chem. 2010, 75, 3268-3273; b) M. Holler, N. Allenbach, J. Sonet, J.-F. Nierengarten, Chem. Commun. 2012, 48 , 2576-2578.

[24] A. N. Kursunlu, C. Baslak, Tetrahedron Lett. 2018, 59, 1958-1962. 


\section{Entry for the Table of Contents}

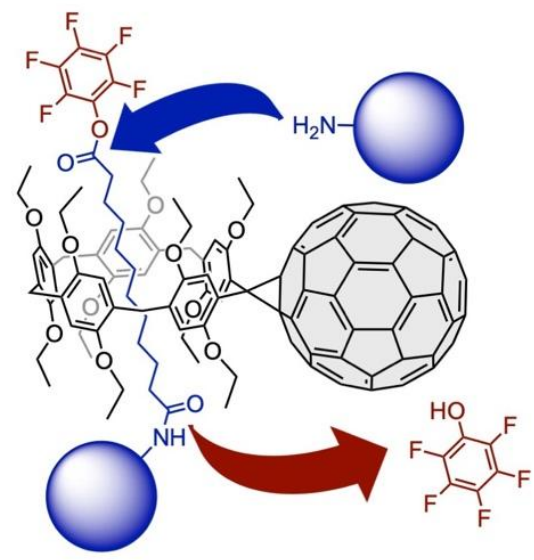

Pillar[5]arene-containing [2]rotaxane building blocks bearing pentafluorophenyl ester functions have been prepared and stopper exchange reactions carried out to generate a wide range of [2]rotaxanes with amide, ester or thioester stoppers. Starting from a fullerene-functionalized pillar[5]arene derivative, this new strategy gave easy access to a photoactive [2]rotaxane incorporating a $\mathrm{C}_{60}$ moiety and two Bodipy stoppers.

Institute and/or researcher Twitter usernames: @INC_CNRS - @Unistra - @LIMA_UMR7042 (Institutions) / @IwonaNieren @nierengarten6 (Researchers). 